\title{
Image Compression using Cosine - Slant Hybrid Wavelet Transform with Assorted Color Spaces
}

\author{
Sudeep D. Thepade,Ph.D \\ Dean (R\&D) \\ Pimpri Chinchwad College of Engineering \\ Pune, India
}

\author{
Jaya $\mathrm{H}$. Dewan \\ Assistant Professor \\ Pimpri Chinchwad College of Engineering \\ Pune, India
}

\begin{abstract}
Gigantic size of multimedia data and images being generated stored and transmitted across the digital web today, which has raised new research concerns in computing field. One of such concerns is compression of such data with minimal quality distortion. In recent work, the hybrid wavelet transforms (HWT) are proven to be better in image compression as compared to the constituent transforms considered individually $[1,2]$. Then further the proportion wise performance comparison of the constituent transforms considered for generation of HWT is also studied [3, 4]. Here the paper presents the performance appraise of various color spaces along with the conventional RGB color space in image compression using cosine-slant HWT with varying proportions of constituent cosine and slant transforms. The experimentation is done on a test-bed having 15 images of various sizes and varied compression ratios. The results show that LUV color space with 1:4 proportion of cosine-slant transform in HWT gives better quality of compression for higher compression ratios (90\% and 95\%). For 85\% compression ratio, LUV color space with 1:1 proportion of cosine-slant transforms in HWT gives better compression quality. While in lower compression ratios (60\% to $80 \%)$, the RGB color space gives better compression with $1: 1$ proportion of cosine-slant transforms in HWT.
\end{abstract}

\section{General Terms}

Image Compression.

\section{Keywords}

Image Compression, Hybrid Wavelet Transforms, Slant Transforms, Discrete Cosine Transform (DCT), Color Spaces.

\section{INTRODUCTION}

Today, due to advances in technology, millions of images are getting generated every hour thus storage and transmission of the images are becoming key issues. Image compression plays an essential role for storage and transmission of images as it reduces the size of images so that images can be stored in less space and less bandwidth is required for transmission [5]. For applications like medical imaging, iris recognition, and finger print recognition, the compression technique must be loss less so that the original image can be regenerated without any loss of information from the compressed image. The applications like web application, mobile applications, weather forecasting can tolerate some amount of image data loss if the decompressed image is not exact as original image. This kind of compression technique is called as lossy. Lossy compression techniques provide high degree of compression as compared to lossless compression techniques. In this paper lossy compression technique in transformed domain is proposed. Previous work has proved that wavelet transforms generated from orthogonal transforms give high degree of compression as compared to original orthogonal transforms $[6,7,8]$. Later work proves that hybrid wavelet transform (HWT) generated using two different orthogonal transforms gives better compression ratio(CR) as compared to orthogonal transforms and wavelet transform generated from its orthogonal transform $[1,2,3,4]$. Here the effect of HWT generated by varying proportions of cosine - slant constituent transforms for image compression with various color spaces is analyzed.

\section{GENERATION OF HYBRID WAVELET TRANSFORM [2]}

The hybrid wavelet transform $\mathrm{T}_{\mathrm{PQ}}$ of size $\mathrm{NxN}$ can be generated by using two orthogonal transforms, say $\mathrm{P}$ and $\mathrm{Q}$ respectively as given in equation(1) and (2) where $\mathrm{N}=\mathrm{a} * \mathrm{~b}=\mathrm{ab}$. The first $b$ rows of HWT matrix are calculated as the product of each element of first row of the orthogonal transform $\mathrm{P}$ with each of the columns of the orthogonal transform Q. For next ' $b$ ' number of rows of HWT matrix, the second row of the orthogonal transform matrix $\mathrm{P}$ is shift rotated after being appended with zeros as shown in equation (2). Similarly the other rows of HWT matrix are generated (as set of b rows each time for each of the ' $a-1$ ' rows of orthogonal transform matrix $P$ starting from second row up to last row).

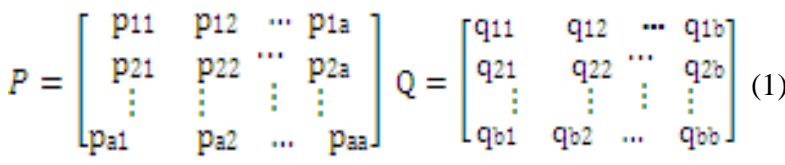

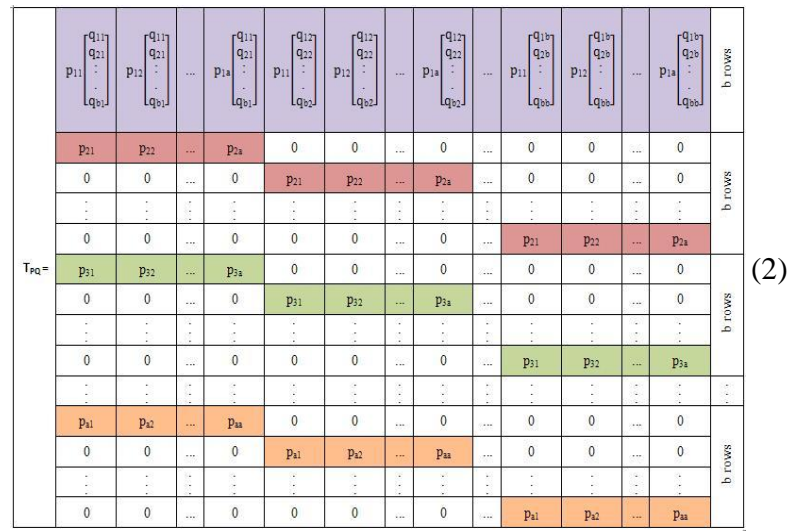




\section{EXPERIMENTATION}

The experimentation of the image compression using cosine slant HWT with assorted color spaces is done with MATLAB 7.14 using a computer with Intel Core i3 Processor2.9 GHz and 4 GB RAM. The color spaces used are RGB, LUV, YUV, $\mathrm{YCbCr}, \mathrm{YCgCb}$, YIQ and XYZ [9]. The test bed used in the experimentation is created by taking 15 different images by with $256 \times 256 \times 3$ and $64 \times 64 \times 3$ sizes as shown in figure 1 . On each image the hybrid wavelet transform generated using cosine transform [10] and slant transform [11] as constituent transform is applied by varying proportion of both the constituent transforms. The image is first converted to the required color space. Then on each image, cosine-slant HWT is applied and the image is compressed in transformed domain. To analyse the performance of HWT with various color spaces, the compressed image is transformed back to spatial domain by applying inverse HWT and converted back to RGB color space. Mean square error (MSE) is calculated between original and modified image for comparing the performance of image compression using cosine-slant HWT with assorted color spaces.

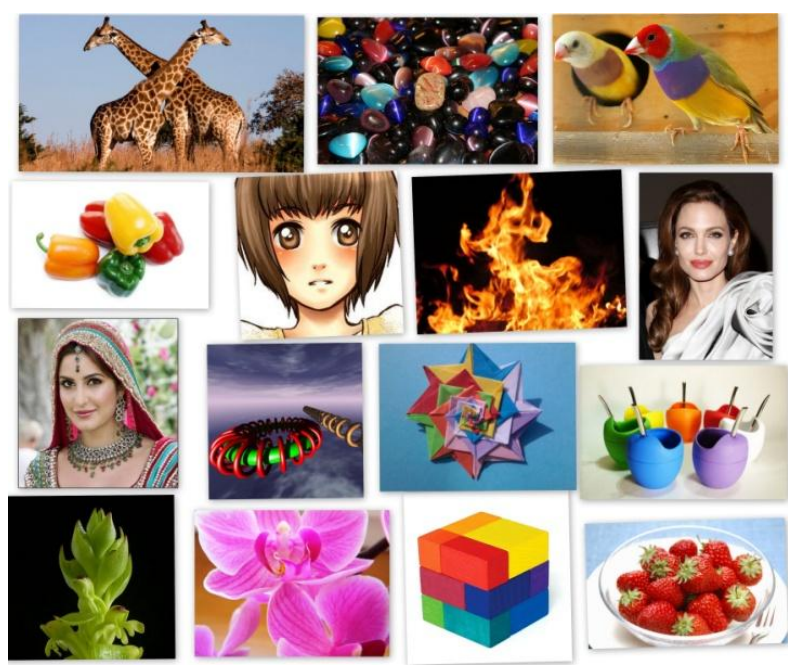

Fig 1:Testbed Used

Eight compression ratios $(60 \%, 65 \%, 70 \%, 75 \%, 80 \%, 85 \%$, $90 \%$ and $95 \%$ ) are considered for performance comparison of color spaces in cosine-slant HWT based image compression. The proportions of cosine and slant constituent orthogonal transforms in HWT are varied and the effects are analysed. The average MSE for each compression ratio, image size and various proportions of cosine-slant constituent transforms in hybrid wavelet transform are considered for performance analysis.

\section{RESULTS AND DISCUSSION}

The results are compared by calculating mean square error between original image and compressed image. The images are varied by various images, size of image; compression ratios, proportion of cosine-slant transform used in generation of hybrid wavelet transform and the color spaces.

Table 1 shows the average mean square error (MSE) between original image and compressed image calculated by varying the images and size of images using LUV color space. The MSEs are calculated for compression ratios of $95 \%, 90 \%$, $85 \%, 80 \%, 75 \%, 70 \%, 65 \%$ and $60 \%$. The ratio of cosine transform and Slant transform is varied as 1:16, 1:4, 1:1, 4:1, and 16:1 in the generation of hybrid wavelet transform.
The HWT generated using 4:1 proportion of cosine-slant transform gives less average MSE value for $95 \%$ and $90 \%$ compression ratios. For compression ratios between $60 \%$ and $85 \%$, HWT generated from 1:1 proportion of cosine-slant constituent transforms gives less average MSE values.

Table 1: Average MSE vs Compression Ratio for all the Images by varying proportion of Cosine-Slant Transform in HWT using LUV Color Space

\begin{tabular}{|c|c|c|c|c|c|c|c|c|}
\hline LUV & $\mathbf{9 5 \%}$ & $\mathbf{9 0 \%}$ & $\mathbf{8 5 \%}$ & $\mathbf{8 0 \%}$ & $\mathbf{7 5 \%}$ & $\mathbf{7 0 \%}$ & $\mathbf{6 5 \%}$ & $\mathbf{6 0 \%}$ \\
\hline Slant & 308.7 & 179.3 & 119.7 & 84.2 & 60.8 & 44.4 & 32.5 & 23.8 \\
\hline $\begin{array}{c}\text { 1:16 } \\
\text { (DCT:Slant) }\end{array}$ & 320.3 & 178.4 & 113.2 & 75.2 & 51.0 & 34.9 & 23.8 & 16.1 \\
\hline $\begin{array}{c}\text { 1:4 } \\
\text { (DCT:Slant) }\end{array}$ & 278.5 & 136.9 & 78.7 & 48.1 & 30.4 & 19.5 & 12.5 & 8.1 \\
\hline $\begin{array}{c}\text { 1:1 } \\
\text { (DCT:Slant) }\end{array}$ & 229.9 & 108.9 & 62.1 & 38.2 & 24.4 & 15.9 & 10.5 & 6.9 \\
\hline $\begin{array}{c}\text { 4:1 } \\
\text { (DCT:Slant) }\end{array}$ & 214.6 & 106.5 & 63.4 & 40.8 & 27.2 & 18.4 & 12.5 & 8.6 \\
\hline $\begin{array}{c}\text { 16:1 } \\
\text { (DCT:Slant) }\end{array}$ & 228.6 & 120.2 & 74.7 & 49.9 & 34.5 & 24.3 & 17.3 & 12.3 \\
\hline $\begin{array}{c}\text { DCT } \\
\text { [Mime }\end{array}$ & 240.6 & 130.4 & 82.9 & 56.4 & 39.7 & 28.4 & 20.5 & 14.8 \\
\hline
\end{tabular}

[Minimum MSE values for respective ratio of compression are shaded indicating better image compression quality]

Table 2: Average MSE vs Compression Ratio for all the Images by varying proportion of Cosine-Slant Transform in HWT using XYZ Color Space

\begin{tabular}{|c|c|c|c|c|c|c|c|c|}
\hline XYZ & $\mathbf{9 5 \%}$ & $\mathbf{9 0 \%}$ & $\mathbf{8 5 \%}$ & $\mathbf{8 0 \%}$ & $\mathbf{7 5 \%}$ & $\mathbf{7 0 \%}$ & $\mathbf{6 5 \%}$ & $\mathbf{6 0 \%}$ \\
\hline Slant & 383.5 & 235.7 & 165.0 & 122.1 & 92.6 & 70.5 & 54.2 & 42.0 \\
\hline $\begin{array}{c}\text { 1:16 } \\
\text { (DCT:Slant) }\end{array}$ & 402.7 & 242.0 & 162.5 & 114.9 & 83.3 & 60.6 & 43.6 & 31.4 \\
\hline $\begin{array}{c}\text { 1:4 } \\
\text { (DCT:Slant) }\end{array}$ & 367.2 & 193.6 & 118.2 & 75.4 & 50.2 & 33.9 & 22.9 & 15.4 \\
\hline $\begin{array}{c}\text { 1:1 } \\
\text { (DCT:Slant) }\end{array}$ & 304.8 & 152.6 & 91.4 & 58.7 & 39.3 & 26.9 & 18.5 & 13.0 \\
\hline $\begin{array}{c}\text { 4:1 } \\
\text { (DCT:Slant) }\end{array}$ & 278.4 & 146.1 & 90.5 & 61.1 & 42.7 & 30.3 & 21.6 & 15.6 \\
\hline $\begin{array}{c}\text { 16:1 } \\
\text { (DCT:Slant) }\end{array}$ & 292.4 & 158.7 & 103.8 & 72.6 & 52.9 & 39.2 & 28.9 & 21.6 \\
\hline \begin{tabular}{c} 
DCT \\
\hline
\end{tabular} & 304.0 & 174.4 & 115.3 & 81.8 & 60.2 & 45.1 & 34.1 & 26.2 \\
\hline
\end{tabular}

[Minimum MSE values for respective ratio of compression are shaded indicating better image compression quality]

Table 2 shows the average MSE for image compression using HWT generated by varying proportions of cosine- slant constituent transforms for various compression ratios with XYZ color space. The results show that for $95 \%, 90 \%$ and $85 \%$ compression ratios, HWT generated from 4:1 proportion of cosine-slant constituent transforms gives less average MSE. For $60 \%$ to $80 \%$ compression ratios, HWT generated from 1:1 proportion of cosine-slant constituent transform gives better result as compared to HWT generated from other proportions of cosine-slant constituent transforms.

Table 3 shows the average MSE for YCbCr color space using HWT generated by varying proportion of cosine-slant constituent transforms. For $90 \%$ and $95 \%$ compression ratios, HWT generated by $4: 1$ proportion of cosine-slant transforms give better image compression quality. HWT generated from 1:1 proportion of cosine-slant transforms gives better compression quality for $60 \%$ to $85 \%$ compression ratios 
Table 3: Average MSE vs Compression Ratio for all the Images by Varying Proportion of Cosine -Slant Transform in HWT using YCbCr Color Space

\begin{tabular}{|c|c|c|c|c|c|c|c|c|}
\hline $\mathrm{YCbCr}$ & $95 \%$ & $90 \%$ & $85 \%$ & $80 \%$ & $75 \%$ & $70 \%$ & $65 \%$ & $60 \%$ \\
\hline Slant & 314.4 & 183.3 & 122.5 & 86.5 & 62.6 & 45.87 & 33.72 & 24.74 \\
\hline $\begin{array}{l}\text { 1:16 } \\
\text { (DCT: } \\
\text { Slant) }\end{array}$ & 326.7 & 183.0 & 116.5 & 77.8 & 53.0 & 36.32 & 24.83 & 16.83 \\
\hline $\begin{array}{c}1: 4 \\
\text { (DCT: } \\
\text { Slant) }\end{array}$ & 284.8 & 140.5 & 81.0 & 49.4 & 31.2 & 20.09 & 12.94 & 8.33 \\
\hline $\begin{array}{c}1: 1 \\
\text { (DCT: } \\
\text { Slant) } \\
\end{array}$ & 234.4 & 111.5 & 63.7 & 39.3 & 25.2 & 16.49 & 10.81 & 7.10 \\
\hline $\begin{array}{c}4: 1 \\
\text { (DCT: } \\
\text { Slant) }\end{array}$ & 218.8 & 109.0 & 65.0 & 41.8 & 27.9 & 18.93 & 12.93 & 8.83 \\
\hline $\begin{array}{l}\text { 16:1 } \\
\text { (DCT: } \\
\text { Slant) } \\
\end{array}$ & 232.2 & 122.0 & 76.1 & 50.9 & 35.3 & 24.96 & 17.78 & 12.68 \\
\hline DCT & 244.4 & 132.9 & 84.7 & 57.7 & 40.7 & 29.14 & 21.12 & 15.35 \\
\hline
\end{tabular}

[Minimum MSE values for respective ratio of compression are shaded indicating better image compression quality]

Table 4: Average MSE vs Compression Ratio for all the Images by varying proportion of Cosine -Slant Transform in HWT using YCgCb Color Space

\begin{tabular}{|c|c|c|c|c|c|c|c|c|}
\hline YCgCb & $\mathbf{9 5 \%}$ & $\mathbf{9 0 \%}$ & $\mathbf{8 5 \%}$ & $\mathbf{8 0 \%}$ & $\mathbf{7 5 \%}$ & $\mathbf{7 0 \%}$ & $\mathbf{6 5 \%}$ & $\mathbf{6 0 \%}$ \\
\hline Slant & 314.5 & 183.8 & 123.3 & 87.0 & 63.1 & 46.28 & 34.02 & 24.99 \\
\hline $\begin{array}{c}\mathbf{1 : 1 6} \\
\text { (DCT: } \\
\text { Slant) }\end{array}$ & 327.1 & 183.3 & 116.8 & 78.0 & 53.1 & 36.44 & 24.88 & 16.91 \\
\hline $\begin{array}{c}\mathbf{1 : 4} \\
\text { (DCT: } \\
\text { Slant) }\end{array}$ & 285.2 & 140.6 & 81.1 & 49.5 & 31.3 & 20.15 & 12.98 & 8.35 \\
\hline $\begin{array}{c}\text { 1:1 } \\
\text { (DCT: } \\
\text { Slant) }\end{array}$ & 234.5 & 111.4 & 63.7 & 39.2 & 25.1 & 16.39 & 10.79 & 7.12 \\
\hline $\begin{array}{c}\text { 4:1 } \\
\text { (DCT: } \\
\text { Slant) }\end{array}$ & 219.1 & 109.0 & 65.0 & 41.8 & 27.9 & 18.92 & 12.93 & 8.86 \\
\hline $\begin{array}{c}\text { 16:1 } \\
\text { (DCT: } \\
\text { Slant) }\end{array}$ & 233.0 & 122.9 & 76.6 & 51.2 & 35.5 & 25.08 & 17.88 & 12.78 \\
\hline $\begin{array}{c}\text { DCT } \\
\text { [Minimum MSE values for respective ratio of compression are shaded } \\
\text { indicating better image compression quality] }\end{array}$ \\
\hline
\end{tabular}

Table 4 shows the average MSE values generated from HWT by varying the proportions of cosine-slant constituent transforms using $\mathrm{YCgCb}$ color space. HWT generated from 4:1 proportion of cosine-slant transform gives better compression quality for $90 \%$ and $95 \%$ compression ratios. HWT generated from 1:1 proportion of cosine-slant constituent transforms gives minimum average MSE value for compression ratios in the range of $60 \%$ to $85 \%$.

Table 5 shows the average MSE for YUV color space using HWT generated with various proportions of cosine-slant constituent transforms. The HWT generated using 1:1 proportion of cosine- slant constituent transforms give better compression quality for compression ratios between $60 \%$ and $85 \%$. For compression ratios of $90 \%$ and $95 \%$, HWT generated from $4: 1$ proportion of cosine-slant constituent transforms gives less average MSE.

Table 6 shows the performance comparison of image compression techniques using HWT generated from cosine and slant transforms using YIQ color space. Results show that HWT generated from 4:1 proportion of cosine-slant transform gives better compression quality for compression ratios from $90 \%$ and $95 \%$. For remaining compression ratios, HWT generated from 1:1 proportion of cosine-slant transform gives better compression quality as compared to other proportions of cosine - slant transform in HWT.

Table 5: Average MSE vs Compression Ratio for all the Images by varying proportion of Cosine-Slant Transform in HWT using YUV Color Space

\begin{tabular}{|c|c|c|c|c|c|c|c|c|}
\hline YUV & $\mathbf{9 5 \%}$ & $\mathbf{9 0 \%}$ & $\mathbf{8 5 \%}$ & $\mathbf{8 0 \%}$ & $\mathbf{7 5 \%}$ & $\mathbf{7 0 \%}$ & $\mathbf{6 5 \%}$ & $\mathbf{6 0 \%}$ \\
\hline Slant & 391.8 & 263.0 & 203.9 & 168.9 & 145.9 & 129.87 & 118.27 & 109.7 \\
\hline $\begin{array}{c}\text { 1:16 } \\
\text { (DCT: } \\
\text { Slant) }\end{array}$ & 403.6 & 262.7 & 197.9 & 160.6 & 136.9 & 120.96 & 110.04 & 102.5 \\
$\begin{array}{c}\text { 1:4 } \\
\text { (DCT: } \\
\text { Slant) }\end{array}$ & 362.7 & 221.9 & 164.0 & 133.8 & 116.4 & 105.67 & 98.90 & 94.50 \\
$\begin{array}{c}\text { 1:1 } \\
\text { (DCT: } \\
\text { Slant) }\end{array}$ & 313.8 & 194.0 & 147.7 & 124.1 & 110.6 & 102.24 & 96.83 & 93.34 \\
\hline $\begin{array}{c}\text { 4:1 } \\
\text { (DCT: } \\
\text { Slant) }\end{array}$ & 299.0 & 191.8 & 149.0 & 126.6 & 113.2 & 104.60 & 98.84 & 94.95 \\
$\begin{array}{c}\text { 16:1 } \\
\text { (DCT: } \\
\text { Slant) }\end{array}$ & 311.5 & 204.2 & 159.4 & 135.1 & 120.1 & 110.20 & 103.36 & 98.54 \\
\hline DCT & 323.9 & 214.7 & 167.6 & 141.5 & 125.0 & 114.01 & 106.38 & 100.9 \\
\hline
\end{tabular}

[Minimum MSE values for respective ratio of compression are shaded indicating better image compression quality]

Table 6: Average MSE vs Compression Ratio for all the Images by varying proportion of Cosine-Slant Transform in HWT using YIQ Color Space

\begin{tabular}{|c|c|c|c|c|c|c|c|c|}
\hline YIQ & $\mathbf{9 5 \%}$ & $\mathbf{9 0 \%}$ & $\mathbf{8 5 \%}$ & $\mathbf{8 0 \%}$ & $\mathbf{7 5 \%}$ & $\mathbf{7 0 \%}$ & $\mathbf{6 5 \%}$ & $\mathbf{6 0 \%}$ \\
\hline Slant & 324.3 & 193.9 & 134.0 & 98.4 & 75.0 & 58.43 & 46.54 & 37.76 \\
\hline $\begin{array}{c}\text { 1:16 } \\
\text { (DCT: } \\
\text { Slant) }\end{array}$ & 338.0 & 194.7 & 128.9 & 90.5 & 66.0 & 49.62 & 38.30 & 30.41 \\
$\begin{array}{c}\text { 1:4 } \\
\text { (DCT: } \\
\text { Slant) }\end{array}$ & 297.4 & 153.6 & 94.1 & 62.8 & 44.7 & 33.67 & 26.63 & 22.10 \\
$\begin{array}{c}\text { 1:1 } \\
\text { (DCT: } \\
\text { Slant) }\end{array}$ & 247.6 & 124.2 & 76.8 & 52.5 & 38.7 & 30.04 & 24.49 & 20.88 \\
\hline $\begin{array}{c}\text { 4:1 } \\
\text { (DCT: } \\
\text { Slant) }\end{array}$ & 230.6 & 121.2 & 77.5 & 54.7 & 41.0 & 32.22 & 26.38 & 22.40 \\
$\begin{array}{c}\text { 16:1 } \\
\text { (DCT: } \\
\text { Slant) }\end{array}$ & 242.9 & 133.6 & 88.2 & 63.4 & 48.1 & 37.91 & 30.96 & 26.00 \\
\hline DCT & 255.1 & 144.4 & 96.7 & 70.1 & 53.4 & 42.1 & 34.2 & 28.6 \\
\hline
\end{tabular}

[Minimum MSE values for respective ratio of compression are shaded indicating better image compression quality] 
Table 7: Average MSE vs Compression Ratio for all the Images by varying proportion of Cosine -Slant Transform in HWT using RGB Color Space

\begin{tabular}{|c|c|c|c|c|c|c|c|c|}
\hline RGB & $\mathbf{9 5 \%}$ & $\mathbf{9 0 \%}$ & $\mathbf{8 5 \%}$ & $\mathbf{8 0 \%}$ & $\mathbf{7 5 \%}$ & $\mathbf{7 0 \%}$ & $\mathbf{6 5 \%}$ & $\mathbf{6 0 \%}$ \\
\hline Slant & 309.7 & 179.9 & 120.0 & 84.3 & 60.8 & 44.41 & 32.57 & 23.83 \\
\hline $\begin{array}{c}\text { 1:16 } \\
\text { (DCT: } \\
\text { Slant) }\end{array}$ & 321.6 & 179.1 & 113.7 & 75.6 & 51.4 & 35.07 & 23.85 & 16.11 \\
\hline $\begin{array}{c}\text { 1:4 } \\
\text { (DCT: } \\
\text { Slant) }\end{array}$ & 279.4 & 137.0 & 78.6 & 48.0 & 30.2 & 19.30 & 12.38 & 7.92 \\
$\begin{array}{c}\text { 1:1 } \\
\text { (DCT: } \\
\text { Slant) }\end{array}$ & 230.1 & 108.9 & 62.2 & 38.2 & 24.4 & 15.82 & 10.35 & 6.77 \\
\hline $\begin{array}{c}\text { 4:1 } \\
\text { (DCT: } \\
\text { Slant) }\end{array}$ & 216.4 & 107.4 & 63.8 & 40.9 & 27.2 & 18.40 & 12.52 & 8.53 \\
\hline $\begin{array}{c}\text { 16:1 } \\
\text { (DCT: } \\
\text { Slant) }\end{array}$ & 228.3 & 120.3 & 75.0 & 50.0 & 34.5 & 24.33 & 17.31 & 12.33 \\
\hline DCT & 241.3 & 130.7 & 83.3 & 56.8 & 39.9 & 28.66 & 20.69 & 14.95 \\
\hline
\end{tabular}

[Minimum MSE values for respective ratio of compression are shaded indicating better image compression quality]

Table 7 shows the performance comparison of image compression technique in RGB color space using cosine transform, slant transform and HWT generated by various proportions of cosine-slant constituent transforms. For 90\% and above compression ratios, HWT generated using 4:1 proportion of cosine-slant constituent transforms gives minimum average MSE value. For compression ratios below $90 \%$, HWT generated from 1:1 proportion of cosine-slant transform gives less average MSE value.

Fig 2 shows the average mean square error (MSE) for 95\% and $90 \%$ compression ratios between original image and respective compressed image with assorted color spaces. The results prove that the HWT using 4:1 proportion of cosineslant transform gives less MSE in case of compression ratios of $90 \%$ and $95 \%$ for all the color spaces. The HWT generated using 4:1 proportion of cosine-slant transform gives minimum MSE with LUV color space for $90 \%$ and $95 \%$ compression ratios as compared to all other color spaces including RGB color space.

Fig 3 shows the average mean square error (MSE) differences between original images and respective compressed images for compression ratios from $85 \%$ to $75 \%$. In case of $85 \%$, $80 \%$ and $75 \%$ compression ratios, HWT generated from 1:1 proportion of cosine-slant transform gives less average MSE. The image compression quality of HWT using 1:1 proportion of cosine-slant transform with LUV color space is better as compared to all other color spaces for $85 \%$ compression ratio. For compression ratios of $75 \%$ and $80 \%$, HWT generated from 1:1 proportion of cosine-slant transform with RGB color space gives minimum average MSE.

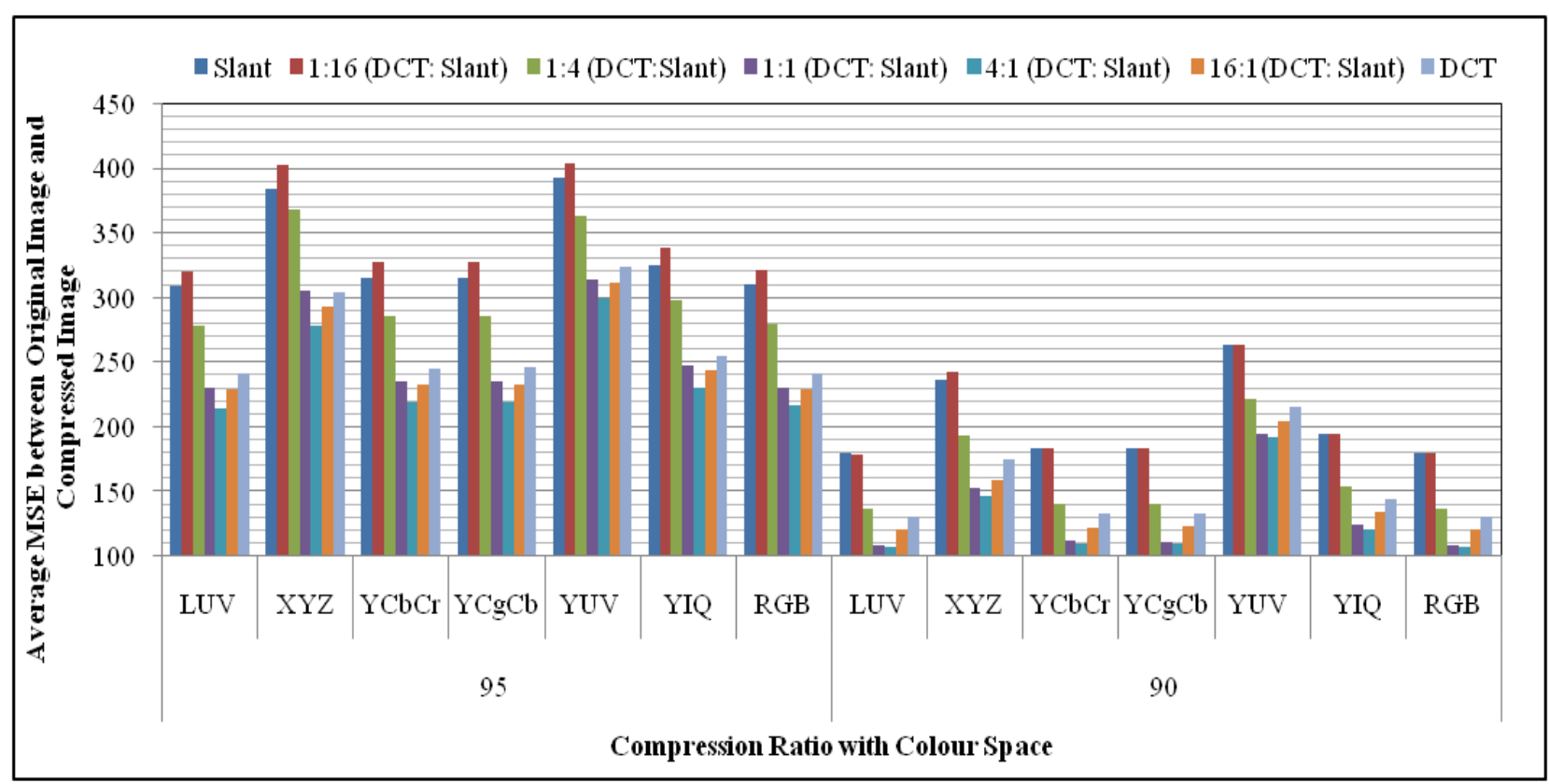

Fig 2:Performance comparison of Image compression for all images by varying proportion of Cosine-Slant transform in HWT with respect to $95 \%$ and $90 \%$ compression ratio for various color spaces

Fig 4 shows the performance comparison of various proportions of cosine-slant transform in HWT with assorted color spaces for the compression ratios of $70 \%, 65 \%$ and $60 \%$. The HWT generated from 1:1 proportion of cosine-slant transform gives better image compression quality with all the color spaces. The best performance is given by $1: 1$ proportion of cosine-slant transform in HWT with RGB color space. 


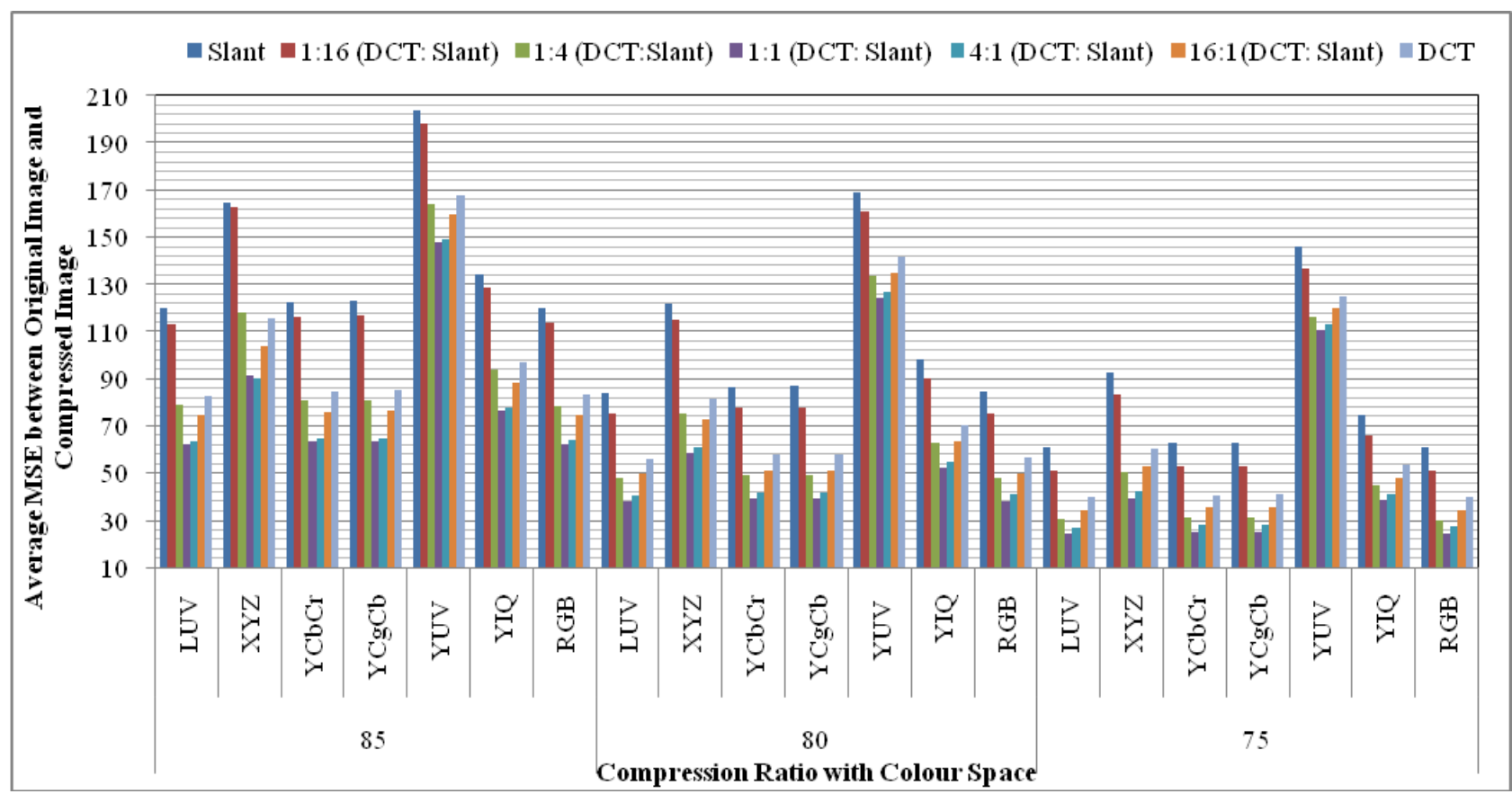

Fig 3: Performance comparison of Image compression for all images by varying proportion of Cosine - Slant transform in HWT with respect to $85 \%$ to $75 \%$ compression ratios for various color spaces

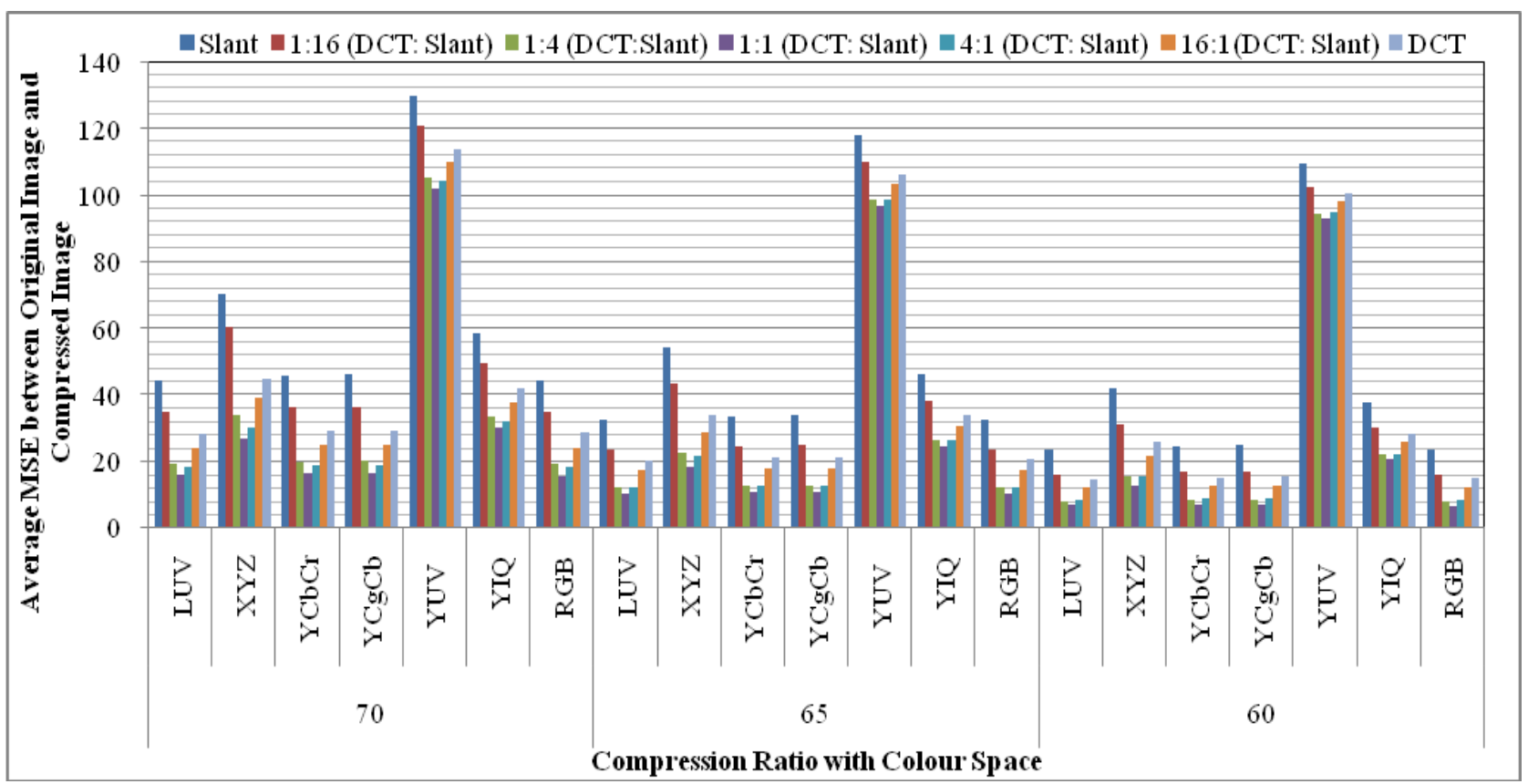

Fig 4: Performance comparison of Image compression for all images by varying proportion of Cosine- Slant transform in HWT with respect to $70 \%$ to $60 \%$ compression ratio using various color spaces

Fig 5 shows the paper-art image compression with compression ratio of $90 \%$ and $95 \%$ using HWT with $1: 4$ proportion of cosine-slant transform. The mean square error is less for LUV color space with respect to RGB color space.

Fig 6 shows the image compression of paper-art's image using 1:1 proportion of cosine-slant constituent transform in HWT for $85 \%$ compression ratio. The subjective quality of compressed image is better with LUV color space than RGB color space. Also, the MSE value using LUV color space is less as compared to RGB color space.

Fig 7 and fig 8 shows the effect of image compression on Strawberry's image for $60 \%$ to $80 \%$ compression ratio using HWT generated from 1:1 proportion of cosine-slant transform. The image is of size $64 \times 64 \times 3$. The MSE with RGB color space is less than MSE with LUV color space. Also, the subjective quality of image compression with RGB color space is better than LUV color space. 


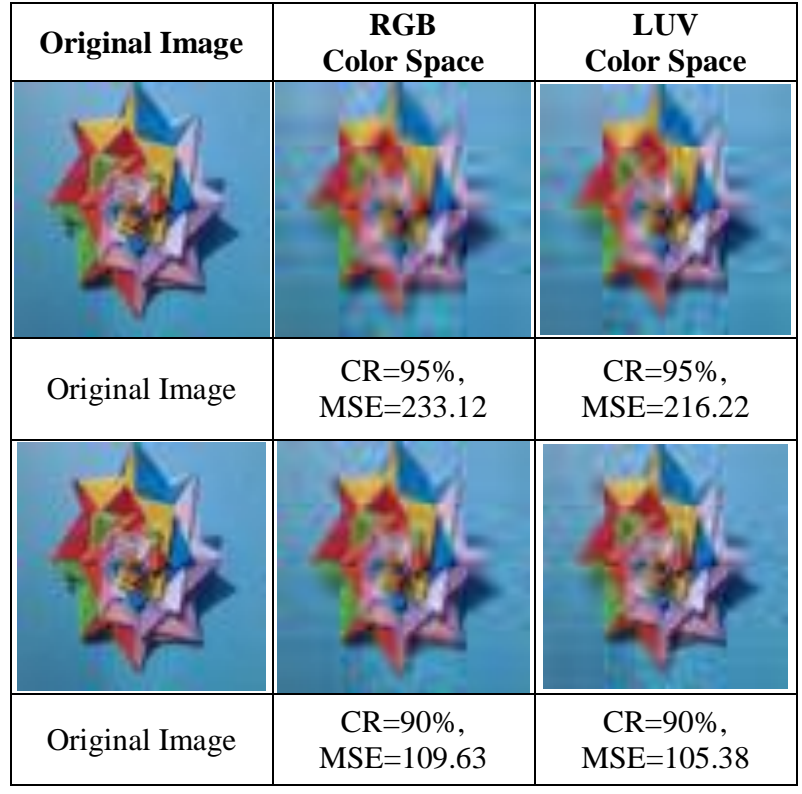

Fig 5: Paper-art's Image Compression using HWT generated with 1:4 proportion of Cosine-Slant transform

\begin{tabular}{|c|c|c|}
\hline Original Image & $\begin{array}{c}\text { RGB } \\
\text { Color Space }\end{array}$ & $\begin{array}{c}\text { LUV } \\
\text { Color Space }\end{array}$ \\
\hline & & \\
& & \\
\hline Original Image & $\begin{array}{c}\mathrm{CR}=85 \%, \\
\mathrm{MSE}=55.67\end{array}$ & $\begin{array}{c}\mathrm{CR}=85 \%, \\
\mathrm{MSE}=51.13\end{array}$ \\
\hline
\end{tabular}

Fig 6: Paper-art's Image Compression using HWT generated with 1:1 proportion of Cosine-Slant transform

\begin{tabular}{|c|c|c|}
\hline Original Image & $\begin{array}{c}\text { RGB } \\
\text { Color Space }\end{array}$ & $\begin{array}{c}\text { LUV } \\
\text { Color Space }\end{array}$ \\
\hline & & \\
\hline Original Image & CR=80\%, & CR=80\%, \\
\hline & MSE=81.58 & MSE 84.63 \\
\hline & & \\
\hline & & \\
\hline
\end{tabular}

Fig 7: Strawberry's Image Compression using HWT generated with 1:1 proportion of Cosine-Slant transform for $75 \%$ and $80 \%$ compression ratio

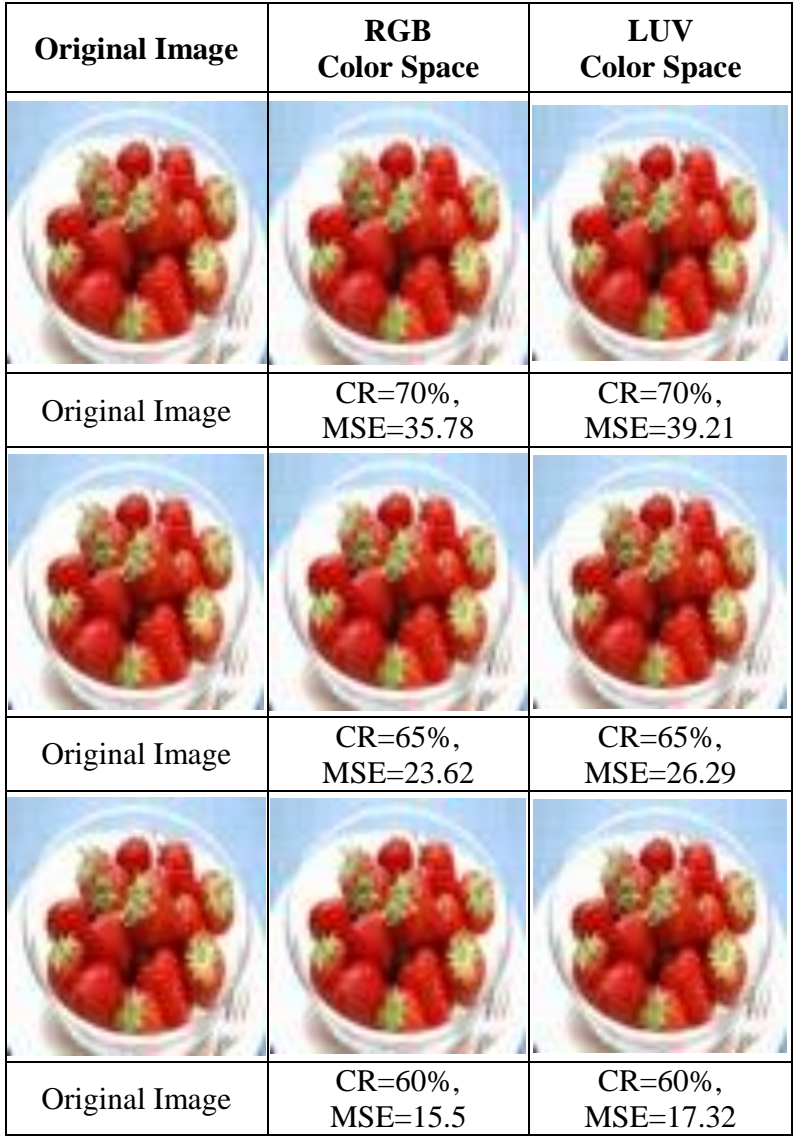

Fig 8: Strawberry's Image Compression using HWT generated with 1:1 proportion of Cosine-Slant transform for $60 \%$ to $70 \%$ compression ratios

\section{CONCLUSIONS}

The cosine-slant HWT is used for image compression with varied proportions of constituent cosine and slant transforms using seven assorted color spaces alias RGB, LUV, YUV, $\mathrm{YIQ}, \mathrm{YCgCb}, \mathrm{YCbCr}$ and $\mathrm{XYZ}$. The experimentation is done with the test-bed of 15 images for various compression ratios from $60 \%$ to $95 \%$. Results have shown that the LUV color space outperforms other color spaces giving better quality of image compression with 1:4 proportion of cosine-slant transform in HWT for $90 \%$ and $95 \%$ compression ratios and 1:1 proportion of cosine-slant transform in HWT for $85 \%$ compression ratio. In case of compression ratios below $85 \%$ RGB color space gives better compression quality with $1: 1$ proportion of cosine-slant transforms in HWT.

\section{REFERENCES}

[1] Dr. H. B. Kekre, Dr. Tanuja K. Sarode, Dr. Sudeep D.Thepade, Ms. Sonal Shroff, "Instigation of Orthogonal Wavelet Transforms using Walsh, Cosine, Hartley, Kekre Transforms and their use in Image Compression", International Journal of Computer Science and Information Security (IJCSIS), Volume 9, Number 6, pp.125-133, June2011 (ISSN: 1947-5500), Available online at http://sites.google.com/site/ijcsis

[2] Dr. H.B.Kekre, Dr.Tanuja K. Sarode, Sudeep D. Thepade "Inception of Hybrid Wavelet Transform using Two Orthogonal Transforms and It's use for Image Compression", International Journal of Computer 
Science and Information Security (IJCSIS), Vol. 9, No. 6,2011

[3] Dr. Sudeep D. Thepade, Jaya H. Dewan "Varying Proportions of Constituent Transforms to Generate Hybrid Wavelet Transform for Image Compression" 1st International Conference on Emerging Trends in computing, communication and Nanotechnology(ICE CCN 2013), Tamilnadu, India. Will be uploaded online at IEEE Xplore.

[4] Dr. Sudeep D. Thepade, Jaya H. Dewan "Extended Performance Comparison of Hybrid Wavelet Transform for Image Compression with Varying Proportions of Constituent Transforms 15th International Conference on Advanced Computing Technologies (ICACT-2013), Rajampet, Andhrapradesh, India. Will be uploaded online at IEEE Xplore.

[5] S.Parveen Banu Dr.Y.Venkataramani, "An Efficient Hybrid Image Compression Scheme based on Correlation of Pixels for Storage and Transmission of Images", International Journal of Computer Applications (0975 - 8887) Volume 18- No.3, March 2011.

[6] Dr. Sudeep Thepade, Shwetali Erandole, "Effect of Tiling in Image Compression using Wavelet Transform for Cosine \& Kekre Transform", International Conference on Emerging Trends in Computing Communication and Nanotechnology, 2013. Will be uploaded on online IEEE Xplore.
[7] Dr.H.B.Kekre, Dipali Sadavarti, Archana Athawale, "Algorithm to Generate Wavelet Transform from an Orthogonal Transform', International Journal Of Image Processing (IJIP), Volume (4): Issue (4).

[8] Dr. Sudeep Thepade, Shwetali Erandole, "Extended Performance Comparison of tiling based Image Compression using Wavelet Transforms \& Hybrid Wavelet Transforms", IEEE International Conference on Energy Efficient Technologies for Sustainability ICEETS-2013. Will be uploaded on online IEEE Xplore.

[9] Dr. H. B. Kekre, Sudeep D. Thepade, Archana Athawale, Adib Parkar, "Using Assorted Color Spaces and pixel window sizes for Colorization of Grayscale images", ACM International Conferences and workshops on emerging Trends in Technology(ICWET 2010), Thakur College of Engg. and Tech.,Mumbai,26-27 Feb 2010.

[10] Rao,K.R. Ahmed, N. "Orthogonal transforms for digital signal processing" IEEE International Conference on Acoustics, Speech, and Signal Processing, ICASSP '76. Vol.1, pp136 - 140.

[11] HouZhengxin, Xu Nini, Chen Hong, Li Xuelei "Fast slant transform with sequency increment and its application in image compression" 7th International Conference on Signal Processing, 2004. Proceedings. ICSP '04. 2004 Vol.1, pp $65-68$. 